\title{
Die spektrale Verschiebung der Emissionsbanden vershieden aktivierter (Zn: Cd) S-Mischkristalluminophore
}

\author{
Von H. Ortmann und H. Treptow \\ Aus der Deutschen Akademie der Wissenschaften zu Berlin, Physikalisch-Technisches Institut, \\ Bereich Lumineszenzforschung, Liebenwalde \\ (Z. Naturforschg. 16 a, 910-916 [1961] ; eingegangen am 4. Mai 1961)
}

Herrn Prof. Dr. N. RieHL zum 60. Geburtstag gewidmet

\begin{abstract}
Es wurden die Cu-, Ag- und eigenaktivierten Luminophore der vollständigen (Zn : Cd)S-Mischkristallreihe aus denselben Grundmaterialien hergestellt und ihre Emission bei langwelliger UVAnregung gemessen. Dabei ergab sich, daß die Bandenverschiebungen bei den verschiedenen Aktivierungen zwar gleichsinnig, aber nicht parallel verlaufen. Der Einfluß der Kristallstruktur auf die jeweilige Lage der Bandenmaxima wird aufgezeigt. Die Bandenverschiebung bei Temperaturerniedrigung ist für die untersuchten Aktivierungen grundsätzlich verschieden, was auf Unterschiede im Zentrenbau hinweist.
\end{abstract}

Die technisch sehr wichtigen (Zn : Cd) S-Luminophore, die eine lückenlose Mischkristallreihe von reinem $\mathrm{ZnS}$ bis zu reinem CdS bilden, zeigen, wenn sie mit $\mathrm{Cu}$ oder $\mathrm{Ag}$ aktiviert sind oder als sogenannte eigenaktivierte Luminophore vorliegen, bei Anregung mit langwelligem UV eine oder mehrere Emissionsbanden. Diese Banden verschieben sich mit zunehmendem CdS-Gehalt stetig nach größeren Wellenlängen. Darüber liegen mehrere Veröffentlichungen vor ${ }^{1-8}$, die neben stark unterschiedlichen Angaben gewisse Unzulänglichkeiten aufweisen. Jeder dieser Autoren untersuchte nur einen Aktivator bei einer Temperatur. Weder im Falle der $\mathrm{Cu}$ - noch der AgAktivierung wurden die Untersuchungen über die gesamte Mischkristallreihe durchgeführt. Eigenaktivierte Luminophore werden in diesen Arbeiten überhaupt nicht erwähnt.

Für den Fall der Ag-Aktivierung wurde von vaN GooL ${ }^{9}$ eine ausführliche Darstellung veröffentlicht. Es wurde nicht nur die allgemein bekannte Ag-Bande über die gesamte Mischkristallreihe gemessen, sondern auch eine nur bei tieferen Temperaturen auftretende und ebenfalls dem Ag zuzuord-

1 S. Asano, Science of Light 4, 32 [1955].

2 W. H. Byler, J. Opt. Soc. Amer. 37, 920 [1947].

3 B. E. Fehr, A. J. Friedman, F. J. Studer u. G. R. Fonda, J. Opt. Soc. Amer. 42, 917 [1952].

4 A.A. Guntz, Ann. de Chim. 5, 157, 963 [1926]; 6, 5 [1926].

5 K. Kamm, Ann. Phys., Lpz. 30, 333 [1937].

6 V. L. Levshin, Zhur. Fiz. Ch. 6, 1277 [1935].

7 S. Rотнsснild, Z. Phys. 108, 24 [1938].

8 S. T. Henderson, Proc. Roy. Soc., Lond. A 173, 323 [1939].

9 W. van Gool, „Halbleiter und Phosphore“, Sammelband d. Vorträge des Internationalen Kolloquiums 1956, S. 602 , Berlin 1958. Philips Res. Rep. 13, 157 [1958].

10 F. A. Kröger u. J. E. Hellingman, J. Electrochem. Soc. 95, 68 [1949]. nende kürzerwellige Bande. Weiterhin wurde erstmalig die Beeinflussung durch die beiden Modifikationen der Kristallstruktur des $\mathrm{ZnS}$ erwähnt.

Aus früheren Arbeiten ${ }^{10-14}$ ist auch bereits bekannt, daß für den Aktivator Cu über ein weites Temperaturgebiet zwei Emissionsbanden auftreten. Unsere Messungen an diesen dem $\mathrm{Cu}$ zuzuordnenden Banden über die gesamte Mischkristallreihe wurden bereits veröffentlicht ${ }^{15}$. Gleichzeitig erschien eine Arbeit von Albers ${ }^{16}$, in der gleichartige Messungen beschrieben wurden. Dabei ist jedoch nur der Verlauf der kurzwelligen Bande bis zum reinen $\mathrm{CdS}$ hin gemessen worden. Der Autor hat beide Banden an denselben Präparaten untersucht, was eine gegenseitige Beeinflussung der Banden nicht ausschließt. Auf die Überlagerung des Effekts der Kristallstruktur, der sich bei den von ihm verwendeten Luminophoren bemerkbar machen mußte, wurde nicht eingegangen.

Wir kennen nur die eine Arbeit von Drosd ${ }^{17}$, in der die Bandenverschiebung der eigenaktivierten ( $\mathrm{Zn}: \mathrm{Cd}) \mathrm{S}$-Luminophore über die gesamte Mischkristallreihe gemessen wurde. Sie ist unseres Wis-

11 E. Grillot u. M. Bancie Grillot, C. R. Acad. Sci., Paris 231, 906 [1950].

12 S. Rothschild, Trans. Faraday Soc. 42, 635 [1946].

13 A. A. Tscherepnew u. T. S. Dobrolubskaja, Dokl. Akad. Wiss., USSR 66, 621 [1949].

14 N. Riehl u. H. Ortmann, Dokl. Akad. Wiss., USSR 66, 6/3, 841 [1949].

15 H. Ortmann u. H. Treptow, „Zur Physik und Chemie der Kristallphosphore". Bericht der Tagung in Greifswald 1959, S. 223, Berlin 1960.

16 K. Albers ${ }^{15}$, ebenda S. 228.

17 L. Drosd, Izv. Akad. Nauk USSR, Serie Fiz. 23,1300 [1959.] 
sens auch die einzige Arbeit, die zwischen der Bandenverschiebung, dargestellt als Frequenz (als Wellenzahl oder Energie) der Maxima über dem molaren $\mathrm{ZnS}$ : CdS-Verhältnis, einen linearen Zusammenhang angibt.

Der Versuch, die Bandenverschiebung für verschiedene Aktivatoren gegenüberzustellen, wurde nur von Leverenz ${ }^{18}$ unternommen. Dabei fehlt jedoch die kurzwellige $\mathrm{Cu}$-Bande. Die langwellige $\mathrm{Cu}$-Bande, die langwellige Ag-Bande und die Bande der Eigenaktivierung konnten nicht für höhere CdSGehalte gemessen werden. Weil sich also die Angaben bis auf ca. 50 Mol-\% beschränken, konnte der vollständige Verlauf der Kurven nicht ausreichend genau erkannt werden.

Unsere umfangreichen Untersuchungen hatten das spezielle Ziel, eventuell auftretende Unterschiede für die drei dicht benachbarten Banden, die kurzwellige $\mathrm{Cu}$-Bande, die langwellige Ag-Bande und die Bande der Eigenaktivierung, festzustellen. Dies wurde besonders durch die nur von Drosp beschriebene lineare Abhängigkeit aktuell. Danach müßte, wenn man alle Veröffentlichungen miteinander vergleicht, zwischen der Fremdaktivierung $(\mathrm{Cu}, \mathrm{Ag})$ und der Eigenaktivierung sich schon äußerlich der Verlauf der Bandenverschiebung durch wesentlich voneinander unterscheidbare Funktionen darstellen lassen. Daraus könnten sich Rückschlüsse auf den Zentrenbau ableiten lassen. Diese Diskrepanz sollte überprüft werden. Um schon kleinste Unterschiede feststellen zu können, müssen die Lage der Bandenmaxima und die dazu gehörenden $\mathrm{ZnS}$ : CdS-Mischungsverhältnisse sehr genau bestimmt werden. Alle uns bekannten Arbeiten mit Ausnahme der von uns bereits veröffentlichten Ergebnisse ${ }^{15}$ enthalten die Abhängigkeiten von dem im Ansatz gewählten Mischungsverhältnis, das die Präparate also vor der Glühung besaßen. Da nach unseren Untersuchungen durch die Glühung beträchtliche Abweichungen zwischen resultierendem und vorher gemischtem $\mathrm{ZnS}$ : CdS-Verhältnis auftreten können, haben wir für sämtliche Proben nach der Glühung das Mischungsverhältnis analysiert und ausschließlich diese Werte für die Darstellung unserer Ergebnisse benutzt. Zur genaueren Bestimmung der Lage der Bandenmaxima wurden die Banden nicht nur bei Zimmertemperatur, sondern auch bei tieferer Temperatur aufgenommen.

18 H.W. Leverenz, An Introduction to Luminescence of Solids, New York 1950.
Bei der Temperatur der flüssigen Luft kann man die Emissionsmaxima wegen der geringeren Bandenbreite deutlicher feststellen.

\section{Präparation der (Zn:Cd)S-Luminophore}

Als Ausgangsmaterial wurde für alle Präparate das von uns selbst gefällte $\mathrm{ZnS}$ und $\mathrm{CdS}$ verwendet. Alle benutzten Ansätze wurden in der Kugelmühle hergestellt, um möglichst homogene Mischungen zu erhalten. Es wurden die folgenden drei verschiedenen Ansätze hergestellt:

$a \mathrm{ZnS}+(1-a) \mathrm{CdS}+2 \% \mathrm{NaCl}+2 \% \mathrm{~S}+10^{-4} \mathrm{~g} \mathrm{Cu} / \mathrm{g}(\mathrm{Zn}: \mathrm{Cd}) \mathrm{S}$,
$a \mathrm{ZnS}+(1-a) \mathrm{CdS}+2 \% \mathrm{NaCl}+2 \% \mathrm{~S}+10^{-4} \mathrm{~g} \mathrm{Ag} / \mathrm{g}(\mathrm{Zn}: \mathrm{Cd}) \mathrm{S}$,
$a \mathrm{ZnS}+(1-a) \mathrm{CdS}+2 \% \mathrm{NaCl}+2 \% \mathrm{~S}$.

Der Zusatz von elementarem Schwefel verhinderte bei der Glühung an Luft die Bildung größerer Mengen von Oxyden. Außerdem wirkt er der S-Fehlstellenbildung entgegen, was dem Auftreten der kurzwelligen $\mathrm{Cu}$-Bande bei der späteren Einwanderung von zusätzlichem $\mathrm{Cu}$ förderlich ist. Die Sulfide der Cu-Reihe, die sich durch Variation der Größe $a$ ergab, wurden 1 Stunde lang bei $1200^{\circ} \mathrm{C}$ geglüht. Dadurch erhielten wir mit rein hexagonaler Struktur kristallisierte Präparate, von denen jedes nur die langwellige Cu-Emissionsbande aufwies. Wegen des hohen Dampfdruckes von $\mathrm{AgCl}$ bei so hohen Temperaturen und dem dabei zu beobachtenden $\mathrm{Ab}$ sinken der Fluoreszenzintensitäten auch bei aktivatorfreien $\mathrm{ZnS}$ - und ( $\mathrm{Zn}: \mathrm{Cd}$ ) S-Luminophoren wurden die entsprechende Ag-Reihe und die aktivatorfreie Reihe bei nur $900{ }^{\circ} \mathrm{C}$ ebenfalls 1 Stunde lang geglüht. Die Ansätze befanden sich in gut abgedeckten Quarztiegeln. Bei den niedrig geglühten Reihen ließen sich durch röntgenographische Untersuchungen bis maximal 15 Mol-\% CdS kubische Reflexe nachweisen. Das CdS selbst besitzt bei Glühtemperaturen über $700{ }^{\circ} \mathrm{C}$ ausschließlich hexagonale Struktur. Obwohl $\mathrm{ZnS}$ bei Temperaturen unter $1020{ }^{\circ} \mathrm{C}$ mit kubischer Struktur (Blende-Typ) kristallisiert, zwingt also das bei der Mischreihe zugefügte CdS ab $15 \%$ auch den bei niedrigen Temperaturen entstehenden Mischkristallen die hexagonale Struktur auf. Um aber über durchgehend hexagonale Mischkristallreihen zu verfügen, wurden zusätzlich für die Ag- und eigenaktivierte Reihe auch noch Präparate bei $1200^{\circ} \mathrm{C}$ geglüht. Als letztes benötigten wir eine Cu-aktivierte Reihe, deren Luminophore nur die kurzwellige $\mathrm{Cu}$-Bande aufwiesen. Hierbei ver- 
fuhren wir folgendermaßen. In einen Teil der Präparate der Cu-aktivierten Reihe wurde zusätzlich $10^{-4} \mathrm{~g} \mathrm{Cu} / \mathrm{g}(\mathrm{Zn}: \mathrm{Cd}) \mathrm{S}$ durch halbstündige Temperung bei $450{ }^{\circ} \mathrm{C}$ eingewandert. Alle so behandelten Präparate zeigten danach keine langwellige, sondern nur noch die kurzwellige Cu-Emissionsbande. Es wurden bewußt beide $\mathrm{Cu}$-Reihen in dieser Weise präpariert, weil dadurch jede gegenseitige Beeinflussung der Banden vermieden wird. Ein weiterer Vorteil dieser Methode liegt darin, daß das Mischungsverhältnis $\mathrm{Zn}: \mathrm{Cd}$ für die lang- und kurzwellige $\mathrm{Cu}$-Reihe identisch ist.

Es standen uns nunmehr vier rein hexagonale Mischkristallreihen zur Verfügung, die jeweils nur eine Emissionsbande zeigten (langwellige Cu-Bande, kurzwellige $\mathrm{Cu}$-Bande, Ag-Bande und eigenaktivierte Bande) und zusätzlich einige Präparate mit geringen CdS-Gehalten, die weitgehend kubisch waren.

\section{Analytische Bestimmung der ZnS:CdS- Mischungsverhältnisse}

Von allen fertig geglühten Präparaten wurde das $\mathrm{ZnS}$ : CdS-Mischungsverhältnis potentiometrisch bestimmt ${ }^{*}$. Diese nachträgliche Bestimmung war notwendig, da die unterschiedlichen Dampfdrucke von $\mathrm{ZnS}$ und $\mathrm{CdS}$ das angesetzte Mischungsverhältnis verändern. Zweitens werden auch die Fehler, die bei Verwendung von nicht vollständig konstant getrockneten Ausgangsmaterialen auftreten, vermieden. Die Abweichungen zwischen dem angesetzten und resultierenden Mischungsverhältnis nach der Glühung betrugen im Durchschnitt $1-2 \mathrm{Mol}-\%$ und konnten in Ausnahmefällen bis zu 5 Mol-\% anwachsen.

\section{Präzisionsbestimmung der Gitterkonstanten}

Um den Zusammenhang zwischen molarem Mischungsverhältnis und den Gitterkonstanten festzustellen, wurden an den rein hexagonalen Präparaten der eigenaktivierten Mischkristallreihe Präzisionsgitterkonstantenbestimmungen nach der Methode von Straumanis durchgeführt. Die Auswertung von je 15 Reflexen unter Berücksichtigung aller auftretenden systematischen Fehler ergab mittels der Aus-

* Einzelheiten der Bestimmungsmethode werden gesondert veröffentlicht.

19 S. Lasof, R. E. Shrader u. H. W. Leverenz, Preparation and Characteristics of Solid Luminescent Materials, Symposium Cornell University 1946, London 1948, S. 214.

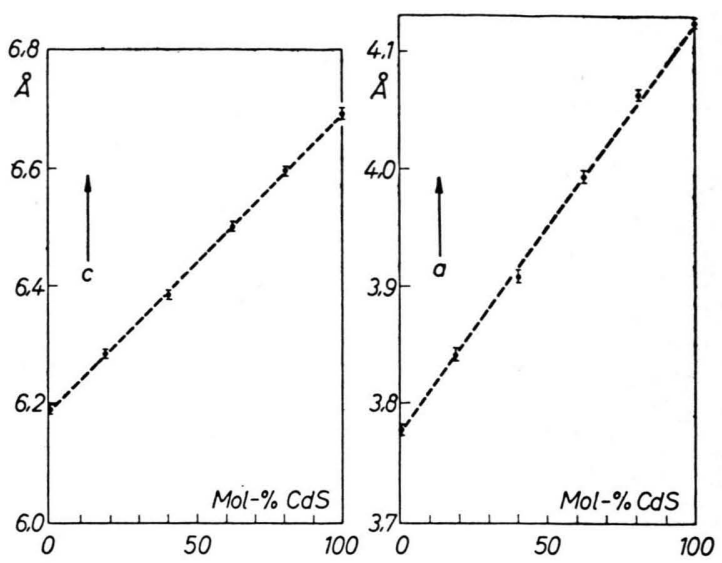

Abb. 1. Darstellung der gemessenen Gitterkonstanten $a$ und $c$ für hexagonale Mischkristalle in Abhängigkeit vom analytisch bestimmten molaren CdS-Gehalt.

gleichsrechnung die in Abb. 1 dargestellten Gitterkonstanten. Auf der Abszisse sind die analytisch bestimmten Mischungsverhältnisse aufgetragen. Der maximale Fehler jeder Gitterkonstantenbestimmung beträgt 2 bis $3 \%$. Innerhalb der Meßgenauigkeit ergibt sich sowohl für $a_{0}$ als auch $c_{0}$ ein linearer $\mathrm{Zu}$ sammenhang. Unsere Messungen stimmen mit denen von LASOF $^{19}$ an synthetischen Luminophoren und Messungen von HuRLbut ${ }^{20}$ an natürlichen Mischkristallen überein. Die unseres Wissens einzigen der WEgardschen Regel widersprechenden Messungen wurden an synthetischen (Zn : Cd) S-Einkristallen durchgeführt ${ }^{21}$. Die von diesen Autoren festgestellten maximalen Abweichungen von $1,4 \%$ können darauf zurückzuführen sein, daß sie zur Bestimmung der ZnS:CdS-Zusammensetzung nicht dieselben Exemplare benutzt haben, wie die zur Messung der Gitterkonstanten.

\section{Untersuchungsmethode der Emissionsbanden}

Die Ausmessung der Emissionsbanden erfolgte bei Zimmertemperatur und bei tiefer Temperatur. Die Kristallpulver wurden im letzteren Falle in eine Vertiefung eines Kupferblockes eingedrückt, der sich auf der Temperatur der flüssigen Luft befand. Welche Temperatur das Kristallpulver selbst hatte, läßt sich nicht genauer angeben. Alle Emissionsbanden

20 C. S. Hurlbut JR., Amer. Mineralogist 42, 184 [1957].

21 N. S. Vitrikhovsкy u. J. B. Mizetskoja, Solid State Phys. 2, 2579 [1960] (russ.). 
wurden ausschließlich bei Anregung mit langwelligem UV untersucht (HBO 200 und Interferenzfilter $365 \mathrm{~nm})$. Zur spektralen Zerlegung der Emission diente ein Zeiß-Monochromator mit Flintglasprisma. Im Spektralgebiet von 400 bis $630 \mathrm{~nm}$ wurde mit einem blauempfindlichen SEV und im Gebiet von 600 bis $1100 \mathrm{~nm}$ mit einem gekühlten, rotempfindlichen SEV, beide von $Z$ e i $ß$, gemessen. Als Vergleichslichtquelle diente eine Wolframbandlampe (BGW). Mit der Energieverteilung des schwarzen Strahlers, dem Emissionsvermögen für Wolfram ${ }^{22}$ und der spektralen Bandbreite des Monochromators wurden die gemessenen Werte auf ein energiegleiches Spektrum umgerechnet und erst dann ausgewertet.

Obwohl der Fehler im blauen Spektralgebiet relativ groß ist, ließ sich durch Verwendung der gleichen Spaltbreite für den Luminophor und die Wolframbandlampe die Lage der Banden für $450 \mathrm{~nm}$ auf $5 \%$ absolut festlegen. Die relative Lage der Banden gegeneinander ist wesentlich genauer feststellbar. Darüber gibt die Reproduzierbarkeit der Meßergebnisse gegeneinander Auskunft. Die Schwankung bei mehrmaligem Messen nimmt von $0,02 \mathrm{eV}$ bei $400 \mathrm{~nm}$ bis zu $0,05 \mathrm{eV}$ bei $630 \mathrm{~nm}$ für den blauempfindlichen SEV zu. Beim gekühlten rotempfindlichen SEV ändert sich die Reproduzierbarkeit von $0,05 \mathrm{eV}$ bei $600 \mathrm{~nm}$ über $0,02 \mathrm{eV}$ bei $850 \mathrm{~nm}$ bis zu $0,05 \mathrm{eV}$ bei $1000 \mathrm{~nm}$. Alle Angaben beziehen sich auf gut meßbare Intensitäten. Nur bei den beiden Präparaten (100 Mol-\% CdS, eigenaktiviert, und 100 Mol-\% CdS : Cu, langwellige Bande), deren Intensitäten sehr gering sind, können die Fehler etwas größer sein. Die durchschnittliche Genauigkeit der relativen Lage der Banden beträgt $0,03 \mathrm{eV}$.

\section{Einfluß der Kristallstruktur auf die Lage der Emissionsbanden}

Wie oben bereits erwähnt, bilden sich je nach der Glühtemperatur bei reinen ZnS-Leuchtstoffen kubische oder hexagonale Kristalle aus, während CdS bei allen von uns verwendeten Glühtemperaturen von rein hexagonaler Struktur war. Nach Untersuchungen von HaRTMANN $^{23}$ erhält man nur für Glühtemperaturen oberhalb $1050{ }^{\circ} \mathrm{C}$ rein hexagonale Struktur für die gesamte Mischkristallreihe. Für niedrige Temperaturen bilden sich bei geringem

22 J. C. DE Vos, Physica 20, 690 [1954].
CdS-Gehalt Mischstrukturen aus, deren RöNtgenDiagramme sowohl die typischen Wurtzit- als auch Blendereflexe, aber mit unterschiedlicher Intensität zeigen. In Abb. 2 sind die Maxima der Emissionsbanden für die Ag-, für die Cu-Aktivierung (langwellige und kurzwellige Bande) und für die Eigenaktivierung in Abhängigkeit von dem Molgehalt an CdS dargestellt. Die ausgezogenen Linien gelten für rein hexagonale Mischkristalle. Die gestrichelten

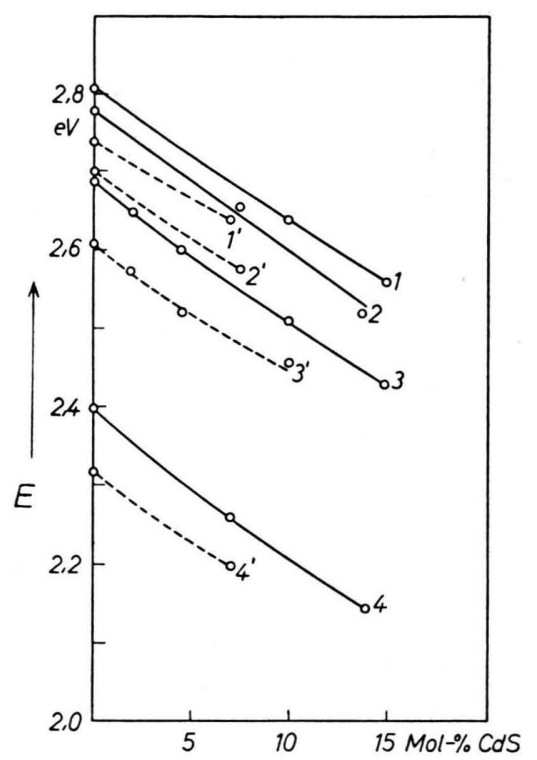

Abb. 2. Strukturbedingte Verschiebung der Emissionsbandenmaxima $(\mathrm{eV})$ in Abhängigkeit vom molaren CdS-Gehalt. 1, 1' Ag-Bande; 2, 2' kurzwellige Cu-Bande; 3, 3' eigenaktivierte Bande; 4, 4' langwellige $\mathrm{Cu}$-Bande; $1,2,3,4$ hexagonale Struktur; $1^{\prime}, 2^{\prime}, 3^{\prime}, 4^{\prime}$ vorwiegend kubische Struktur.

Linien gehen vom rein kubischen $\mathrm{ZnS}$ aus. Mit zunehmendem CdS-Gehalt nimmt dann der hexagonale Bestandteil zu. Bei der von uns verwendeten Glühtemperatur von $900{ }^{\circ} \mathrm{C}$ läßt sich durch die RöNTGENDiagramme ein kubischer Einfluß nur bis maximal 15 Mol-\% CdS nachweisen. Eine Gegenüberstellung der Bandenmaxima für die verschiedene Aktivierung in Abhängigkeit von der Kristallstruktur ergibt die interessante Feststellung, daß die Differenz der Bandenmaxima für die hexagonale und kubische Kristallstruktur innerhalb der Meßgenauigkeit unabhängig von der Art der Aktivierung ist, sie ist nur durch die verschiedene Kristallstruktur bestimmt (Tab. 1).

23 H. Hartmann, Fortschr. Mineralogie 38, 185 [1960]. 


\begin{tabular}{|l|c|c|c|}
\hline Emissionsbande & \multicolumn{2}{|c|}{$\begin{array}{c}\text { Bandenmaximum } \\
\text { in eV } \\
\text { hexagonal kubisch }\end{array}$} & $\begin{array}{c}\text { Differenz } \\
\text { in eV }\end{array}$ \\
\hline Ag aktiviert & 2,81 & 2,74 & 0,07 \\
Cu aktiviert, & 2,78 & 2,70 & 0,08 \\
$\quad$ kurzwellige B. & 2,69 & 2,61 & 0,08 \\
eigenaktiviert & 2,40 & 2,32 & 0,08 \\
\hline $\begin{array}{c}\text { Cu aktiviert, } \\
\text { langwellige B. }\end{array}$ & & & \\
\hline
\end{tabular}

Tab. 1.

\section{Verlagerung der Banden bei Temperatur- änderung}

So wie die verschiedene Kristallstruktur eine doppelte Mannigfaltigkeit für die Lage der Banden ergab, bringt auch die Temperaturänderung von Zimmertemperatur zu tieferen Temperaturen teilweise eine Verschiebung der Banden mit sich. Wir untersuchten alle Präparate bei Zimmertemperatur und in einer Apparatur, die mit flüssiger Luft gekühlt wurde. Welche Temperaturen die Leuchtstoffe dabei annahmen, läßt sich nicht genau angeben, da das thermodynamische Gleichgewicht auch insbesondere von dem unterschiedlichen Absorptionsvermögen der Leuchtstoffe abhängig ist. Daß im Bereich von 0 bis 18 Mol-\% CdS im Ausläufer, für 18 bis 100 Mol-\% CdS im Grundgitter angeregt wird, muß beachtet werden, da alle Untersuchungen bei Anregung mit $365 \mathrm{~nm}$ oder $3,40 \mathrm{eV}$ durchgeführt wurden. Für die 100-proz. ZnS-Leuchtstoffe der vier verschieden aktivierten Mischkristallreihen konnte im Rahmen unserer Meßgenauigkeit für die Ag-Bande und die langwellige $\mathrm{Cu}$-Bande sowohl für die hexagonale als auch kubische Struktur bei Temperaturerniedrigung keine Bandenverschiebung festgestellt werden. Im Gegensatz dazu zeigen die kurzwellige $\mathrm{Cu}$-Bande und die Bande der Eigenaktivierung eine gut meßbare Ver-

\begin{tabular}{|l|l|c|c|c|}
\hline \multirow{2}{*}{$\begin{array}{c}\text { Emissions- } \\
\text { bande }\end{array}$} & Struktur & \multicolumn{2}{|c|}{$\begin{array}{c}\text { Bandenmaximum } \\
\text { in } \mathrm{eV}\end{array}$} & $\begin{array}{c}\text { Differenz } \\
\text { in } \mathrm{eV}\end{array}$ \\
& & $\begin{array}{c}\text { Zimmer- } \\
\text { temp. }\end{array}$ & Temp. & \\
\hline Cu, kurz- & hexa- & & & \\
wellige & gonal & 2,78 & 2,83 & $+0,05$ \\
Bande & kubisch & 2,70 & 2,75 & $+0,05$ \\
eigen- & hexa- & & & \\
aktivierte & gonal & 2,69 & 2,63 & $-0,06$ \\
Bande & kubisch & 2,61 & 2,55 & $-0,06$ \\
\hline
\end{tabular}

Tab. 2.

24 J. H. Gisolf, Physica 6, 84 [1939].

25 G. Wendel u. G. Richter, Z. Phys. Chem. 214, 253 [1960]. schiebung, die zur Unterscheidung dieser beiden Banden benutzt werden kann ${ }^{25}$.

Die Verschiebung verläuft einander entgegengesetzt und ist unabhängig von der Kristallstruktur. Die Ag-Bande und die langwellige $\mathrm{Cu}$-Bande zeigen keine meßbare temperaturabhängige Verschiebung. Das stützt die Annahme, daß es sich dabei um gleichartig gebaute Zentren handelt. Der große Abstand beider Banden $(0,4 \mathrm{eV})$ wäre dann durch den energetischen Unterschied zwischen Ag- und $\mathrm{Cu}$-Ionen zu erklären.

Überzeugender als der wenig stark ausgeprägte Unterschied bei Zimmertemperatur zwischen der AgBande, der kurzwelligen $\mathrm{Cu}$-Bande und der eigenaktivierten Bande deutet das unterschiedliche Verhalten bei Temperaturerniedrigung darauf hin, daß es sich hier um drei prinzipiell verschiedene Zentren handelt. Ihre energetische Lage wird durch die Verringerung der Schwingungsamplitude der Gitterbausteine in Zusammenhang mit der geringfügigen Änderung der Gitterkonstanten grundsätzlich verschieden beeinflußt. Es kann sich kaum um verschiedene Ionen oder Fehlstellen an gleichen Gitter- oder Zwischengitterplätzen handeln, sondern die Zentren müssen an verschiedenen Gitterplätzen lokalisiert sein, z. B. Gitterplatz, Zwischengitterplatz und Fehlstelle.

Mit steigendem CdS-Gehalt nehmen die Verschiebungen, die dann auch nur für die kurzwellige $\mathrm{Cu}$ Bande und eigenaktivierte Bande feststellbar sind, rasch ab und können bereits zwischen 15 und $20 \mathrm{Mol}$ \% CdS nicht mehr festgestellt werden. Die Änderung der Temperatur der untersuchten Pulver, die bei 18 Mol-\% CdS durch den Übergang der Anregung vom Ausläufer in das Grundgitter vor sich geht, kann dieses Verschwinden allein nicht erklären, da sich bei 2 bis 5 Mol-\% CdS die Verschiebung bereits merklich verringert hat. Es scheint sich vielmehr der Anteil von CdS auf diese Effekte negativ auszuwirken, vielleicht in der Weise, daß die Zentren in der direkten Nachbarschaft von Cd-Ionen lokalisiert sind. Um diesen Einfluß näher zu untersuchen, wäre eine weitere Steigerung der Meßgenauigkeit notwendig, was kaum möglich sein wird, da dann alle eingehenden Fehlerquellen nicht mehr mit genügender Exaktheit berücksichtigt werden können.

\section{Die Bandenverschiebung mit steigendem CdS-Gehalt}

In Tab. 3 sind aus den uns bekannten Arbeiten die Daten über die Lage der Emissionsbanden des 
$\mathrm{ZnS}$ und des CdS für verschiedene Aktivierungen zusammengestellt. Dabei wurden nur die bei Zimmertemperatur gemessenen Werte der in hexagonaler Struktur kristallisierten Präparate berücksichtigt. Es zeigt sich völlig unerwartet, daß die von uns beim $\mathrm{ZnS}$ gewählte tabellarische Ordnung mit abnehmender Energie der Emission für das CdS nicht die gleiche Reihenfolge aufweist. Die kurzwellige $\mathrm{Cu}$-Bande ist mit der eigenaktivierten Bande vertauscht (Tab. 3).

\begin{tabular}{|l|c|c|c|c|}
\hline \multirow{2}{*}{ Aktivierung } & \multicolumn{3}{|c|}{ angenäherte Lage der Bandenmaxima } \\
& \multicolumn{3}{|c|}{$\mathrm{ZnS}$} & \multicolumn{2}{c|}{$\mathrm{CdS}$} \\
\cline { 2 - 5 } & $\mathrm{nm}$ & $\mathrm{eV}$ & $\mathrm{nm}$ & $\mathrm{eV}$ \\
\hline $\begin{array}{l}\text { Ag-Bande } \\
\begin{array}{l}\text { Cu, } \\
\text { kurzw. B. } \\
\text { eigen- }\end{array}\end{array}$ & 437 & 2,84 & 730 & 1,70 \\
$\begin{array}{l}\text { aktiv. B. } \\
\begin{array}{l}\text { Cu, } \\
\text { langw. B. }\end{array}\end{array}$ & 470 & 2,64 & 760 & $1,63 *$ \\
\hline
\end{tabular}

* Dieser Wert ist nur aus der Veröffentlichung von DRosD ${ }^{17}$ bekannt; alle anderen Angaben sind dagegen mehreren Arbeiten entnommen.

Tab. 3.

Unsere Messungen ergaben mit maximal $0,03 \mathrm{eV}$ Abweichung Übereinstimmung mit den Angaben für $\mathrm{ZnS}$ und mit maximal $0,05 \mathrm{eV}$ Abweichung Übereinstimmung mit den Angaben für CdS. Nur für die eigenaktivierte Bande von CdS finden wir im Gegensatz zu den Ergebnissen von Drosd ein Emissionsmaximum von $1,56 \mathrm{eV}$. Aus den Werten folgt zwangsläufig, daß sich die Kurven der Bandenverschiebung über die Mischkristallreihe kreuzen müssen. Unsere Meßwerte sind in Abb. 3 dargestellt. Die Lage der Maxima in $\mathrm{eV}$ wurde über dem analytisch bestimmten molaren Gehalt an CdS oder, da nach Abb. 1 ein linearer Zusammenhang zwischen Mol-\% und Gitterkonstanten besteht, über der Gitterkonstanten aufgetragen. Alle Kurven zeigen einen gekrümmten hyperbelähnlichen Verlauf, d. h. die Abhängigkeit der Emissionsenergie ist nicht direkt der Gitterkonstanten proportional. Das ist auch zu erwarten, wenn man bedenkt, daß die röntgenographisch bestimmten Mittelwerte für die Gitterkonstanten nicht mit den Abständen des Zentrums zu den nächsten Gitterbausteinen übereinstimmen. Das Zentrum wird immer eine Verzerrung der idealen Gitterordnung mit sich bringen.

Die Meßwerte für die Ag-Bande stimmen innerhalb der Fehlergrenze mit den Angaben von vas

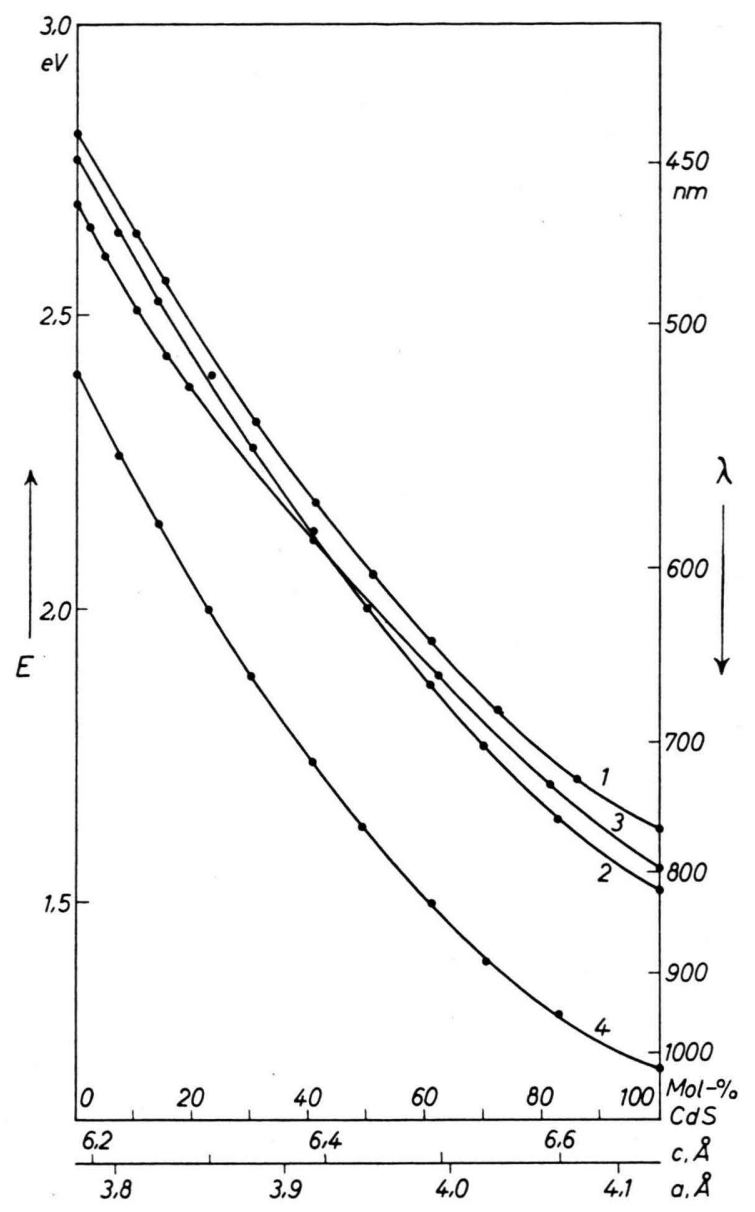

Abb. 3. Darstellung der Bandenverschiebung für hexagonale Mischkristalle in Abhängigkeit vom molaren CdS-Verhältnis oder den Gitterkonstanten $a$ und $c$.

Gool überein ${ }^{9}$. Dagegen zeigen unsere Messungen eindeutig, daß die von Albers ${ }^{16}$ angegebene Parallelität zwischen der $\mathrm{Ag}$ - und den $\mathrm{Cu}$-Banden bzw. den $\mathrm{Cu}$-Banden untereinander nicht bestehen kann. Weiterhin zeigen unsere Messungen, daß auch im Falle der Eigenaktivierung kein grundsätzlicher Unterschied zu den anderen Aktivierungen auftritt. Bei geringen CdS-Gehalten stimmen wir mit den Angaben von DrosD ${ }^{17}$ überein, mit wachsendem CdSGehalt wird die Abweichung dann aber größer.

Es soll sich bei dieser Arbeit vor allem um die Angabe von Daten der Emissionsmaxima mit sehr geringen Fehlern für die meist verwendeten Aktivierungen jedes beliebig zusammengesetzten $(\mathrm{Zn}: \mathrm{Cd}) \mathrm{S}$ Luminophors handeln. Eine fruchtbare Diskussion über Einzelheiten kann nur im Zusammenhang mit dem noch immer nicht geklärten kristallchemischen 
Aufbau der Leuchtzentren geführt werden. Dabei scheint sicher, daß die Art der Kristallbaufehler den Einbau des Aktivators bestimmt. Daraus ergibt sich die Annahme einer größeren Mannigfaltigkeit des Zentrenbaus, die mit den experimentellen Erfahrungen durchaus übereinstimmt. Weitere Aussagen wer- den möglich sein, wenn eine Berechnung des Zusammenhanges zwischen Emissionsenergie und Ausdehnung des Zentrums im gestörten Kristallfeld vorliegt. Die hier aufgezeichneten Meßwerte könnten dann zur Bestätigung solcher Berechnungen herangezogen werden.

\title{
Indium als Koaktivator in $\mathrm{ZnS}$-Cu-Luminophoren
}

\author{
Von G. Wendel \\ Aus der Deutschen Akademie der Wissenschaften zu Berlin, Physikalisch-Technisches Institut; \\ Bereich: Strahlungsquellen \\ (Z. Naturforschg. 16 a, 916-920 [1961]; eingegangen am 3. Mai 1961)
}

Herrn Prof. Dr. N. RieHL zum 60. Geburtstag gewidmet

\begin{abstract}
Indium diffundiert von ca. $500{ }^{\circ} \mathrm{C}$ ab in einen $\mathrm{ZnS}$-Luminophor ein, gibt aber allein keine wesentliche Änderung des Emissionsspektrums. Durch nachträgliches Eindiffundieren von Kupfer entsteht eine breite orange-rote Emission mit einem Maximum bei ca. 6700 Å. Diese Bande kann schon bei kleinen Kupferkonzentrationen von $2 \cdot 10^{-6} \mathrm{~g} \mathrm{Cu} / \mathrm{g} \mathrm{ZnS}$ auftreten. Die notwendige Kupfer- und Indiumkonzentration ist abhängig von der Glühatmosphäre des Ausgangsleuchtstoffes. Die langwellige Emission wird im Gegensatz zur roten Kupferemission, wie sie von F $F_{\text {RoELICH }}$ gefunden wurde, nicht beeinträchtigt durch Anwesenheit von Sauerstoff oder eingebauten Halogen-Ionen.

Auf Grund der Versuche liegt es nahe, diese Emission einem assoziierten Kupfer-Indium-Leuchtzentrum zuzuschreiben.
\end{abstract}

Indium als Koaktivator ist in doppelter Weise interessant für die Diskussion der Lumineszenz der Zinksulfide: Einmal besteht die grundsätzliche Frage der Wirkung der Koaktivatoren, besonders der 3wertigen Kationen; außerdem besteht die Diskussion um die langwellige orange-rote Lumineszenzbande und die damit verbundene Frage des assoziierten Koaktivator-Aktivator-Zentrums.

KRöGER und Mitarb. ${ }^{1}$ haben für die Behandlung der Lumineszenz der Zinksulfide den Begriff des Koaktivators eingeführt. Mit diesem sollte die Wirkung der Halogenide bei der Präparation der Leuchtstoffe erklärt werden. Der Koaktivator soll zur Ladungskompensation dienen und damit auch die Wirkung der 3-wertigen Kationen verständlich machen.

Gegen diese Vorstellung haben sich RIEHL und Ortmans $^{2}$ gewandt, die darauf hinwiesen, daß die Wirkung der Halogenide im wesentlichen in einer Desulfurierung besteht, und da $ß$ das Leuchtzentrum nicht aus dem Aktivator und einem Halogen-Ion, sondern einer Schwefellücke mit Aktivatoren besteht. Die Wirkung der 3-wertigen Kationen als Koakti-

1 F. A. Kröger, J. E. Helingman u. N. W. Smit, Physica 15, 990 [1949]. - B. J. Verwey u. F. A. KRöGER, Philips techn. Rdsch. 13, 90 [1951].

2 N. Riehl u. H. Ortmans, Uber den Aufbau der ZinksulfidLuminophore, Verlag Chemie G.m.b.H., Weinheim 1957.N. RieHL, Halbleiter und Phosphore, Vortr. d. Intern. Kol- vatoren wird in Frage gestellt. Es wird darauf hingewiesen, daß die Glühtemperaturen der Luminophore mit diesen Koaktivatoren zwischen 1100 und $1200{ }^{\circ} \mathrm{C}$ liegen, und es wird vermutet, daß die 3 wertigen Kationen zusätzlich desulfurierend wirken könnten. KRöger und DikofF ${ }^{3}$ haben aber gezeigt, $\mathrm{da} \mathrm{B}$ mit 3-wertigen Kationen, wie $\mathrm{Ga}$, In und Sc, mit den Aktivatoren neue langwellige rotorange Banden im $\mathrm{ZnS}$ entstehen, die sowohl abhängig vom Aktivator wie vom Koaktivator zu sein scheinen. Froelich $^{4}$ fand dagegen eine rote Bande im $\mathrm{ZnS}-\mathrm{Cu}$ bei hohen Kupferkonzentrationen ohne Koaktivator, wenn die Leuchtstoffe in einer $\mathrm{H}_{2} \mathrm{~S}$-Atmosphäre zwischen $1100-1200{ }^{\circ} \mathrm{C}$ geglüht werden. Diese rote Kupferbande ist stark von den Präparationsbedingungen abhängig, bei Glühtemperaturen unter $1000{ }^{\circ} \mathrm{C}$ treten nur die bekannte grüne und blaue Bande auf. Sind kleine Anteile von Sauerstoff vorhanden, kann sich die rote Kupferbande nicht entwickeln.

Diese Ergebnisse wurden durch Untersuchungen von Aven und Potter ${ }^{5}$, ferner von van Gool und

loquiums 1956 in Garmisch-Partenkirchen, BraunschweigBerlin 1958.

3 F. A. Kröger u. J. Dikhoff, Physica 16, 297 [1950].

${ }^{4}$ H. C. Froelich, J. Electrochem. Soc. 100, 280, 496 [1953].

5 M. H. Aven u. R. M. Potter, J. Electrochem. Soc. 105, 134 [1958]. 